\title{
Mapping Ferroelectricity in Hafnia Thin Films with STEM EBIC
}

Ho Leung Chan ${ }^{1}$, William A. Hubbard ${ }^{1}$, Jared J. Lodico ${ }^{1}$ and B. C. Regan ${ }^{1 *}$

${ }^{1 .}$ Department of Physics \& Astronomy and California NanoSystems Institute, University of California, Los Angeles, CA, USA.

* Corresponding author: regan@physics.ucla.edu

Since the recent, unexpected discovery of ferroelectricity in hafnia $\left(\mathrm{HfO}_{2}\right)$ [1], great effort has been put into understanding this phenomenon in this material. Hafnia's ferroelectric behavior is thought to emerge from a non-equilibrium polar orthorhombic $\mathrm{Pca}_{2}$ phase. This phase's unit cell has two stable states that are switchable by an external electric field [3]. However, conclusively identifying the source of ferroelectricity in hafnia is challenging for several reasons: (1) the ferroelectric phase seems to only be stabilized in films thinner than $30 \mathrm{~nm}$, (2) field cycling can drive structural phase transitions, and (3) the films are polycrystalline and invariably consist of multiple phases [4]. Furthermore, the roles that grain structure and heterointerfaces play in stabilizing or destabilizing ferroelectricity remain a mystery [5].

Here, we map hafnia's transition into its ferroelectric phase with traditional scanning transmission microscopy (STEM) and electron beam-induced current imaging (STEM EBIC). In EBIC imaging, currents induced in the sample by the rastering STEM beam are measured by a transimpedance amplifier and associated, pixel by pixel, with the beam position to form an image. EBIC imaging is commonly used to map electric fields, as electron-hole pairs created by the incident beam split up in local electric fields to produce large, easily detected currents $[6,7]$.

Devices are fabricated on an electron transparent $\mathrm{Si}_{3} \mathrm{~N}_{4}$ membrane with an e-beam patterned Ti/Pt $(5 / 25$ $\mathrm{nm}$ ) heater (Fig. 1A and B). The heater is encapsulated in $7 \mathrm{~nm}$ of hafnia and $20 \mathrm{~nm}$ of alumina, both deposited conformally via atomic layer deposition. Finally, a $30 \mathrm{~nm}$ amorphous carbon film is evaporated. Attaching this film to a transimpedance amplifier holds it at virtual ground and produces an electrontransparent top electrode from which the EBIC is collected. Any polarization in the hafnia film produces a local electric field that will separate electron-hole pairs produced by the beam. Thus the EBIC maps contain information about the local ferroelectric properties of the hafnia/alumina stacks.

In Fig. 1C-H, a hafnia device is heated and transitioned into its ferroelectric phase in situ. Figure $1 \mathrm{C}$ and $\mathrm{D}$ show a device before the transition took place. Prior to acquiring $\mathrm{C}$ and $\mathrm{D}, 2 \mathrm{~V}$ was applied between the entire heater (no heater current flowing) and the carbon top electrode, and then this voltage was taken to $0 \mathrm{~V}$. The EBIC signal in Fig. 1D appears mostly grey along the heater, indicating no polarization persisted once the bias voltage was removed. Upon energizing the heater, crystallization was observed in the hafnia thin film in the vicinity of the heater (Fig. 1E). EBIC acquired after heating (Fig. 1F) also shows no sign of ferroelectricity; however, evidence of a persistent electric field can be seen after a bias of $2 \mathrm{~V}$ was again applied to the (cold) heater and removed (Fig. 1G). Along portions of the heater within the crystallized region, Fig. $1 \mathrm{G}$ (and, more obviously, the difference image $\mathrm{H}$ ) shows bright EBIC signal which is indicative of an electric field pointing from the heater to the carbon top electrode.

Applying a bias voltage between the heater and the carbon top electrode prior to crystallization did not polarize the hafnia thin film. After recrystallization via heating, a persistent electric field only appeared once a bias voltage was applied and only in recrystallized regions. These two pieces of evidence indicate 
that 1) applying and then removing an external electric field produced a persistent polarization within the hafnia, and 2) this effect appeared only if the region was previously crystallized via heating. This evidence supports the theory that ferroelectricity originates from hafnia's crystal structure. Further correlation of ferroelectric regions (as indicated by EBIC) with the hafnia crystal structure (as determined with traditional STEM) can provide details about how the different phases contribute to this behavior [8].

\section{References:}

[1] TS Böscke et al., Applied Physics Letters 99 (2011), p. 102903.

[2] J Müller et al., ECS Journal of Solid State Science and Technology 4 (2015), p. N30.

[3] T Mikolajick et al., MRS Bulletin 43 (2018), p. 340.

[4] S Migita, ECS Transactions 80 (2017), p. 247.

[5] ED Grimley, Advanced Materials Interfaces 5 (2018), p. 1701258.

[6] ER White et al., Applied Physics Letters 107 (2015), p. 223104.

[7] WA Hubbard et al., Physical Review Applied 10 (2018), p. 044066.

[8] This work was supported by National Science Foundation (NSF) award DMR-1611036, by NSF Science and Technology Center (STC) award DMR-1548924 (STROBE), and by the UCLA PSEIF.
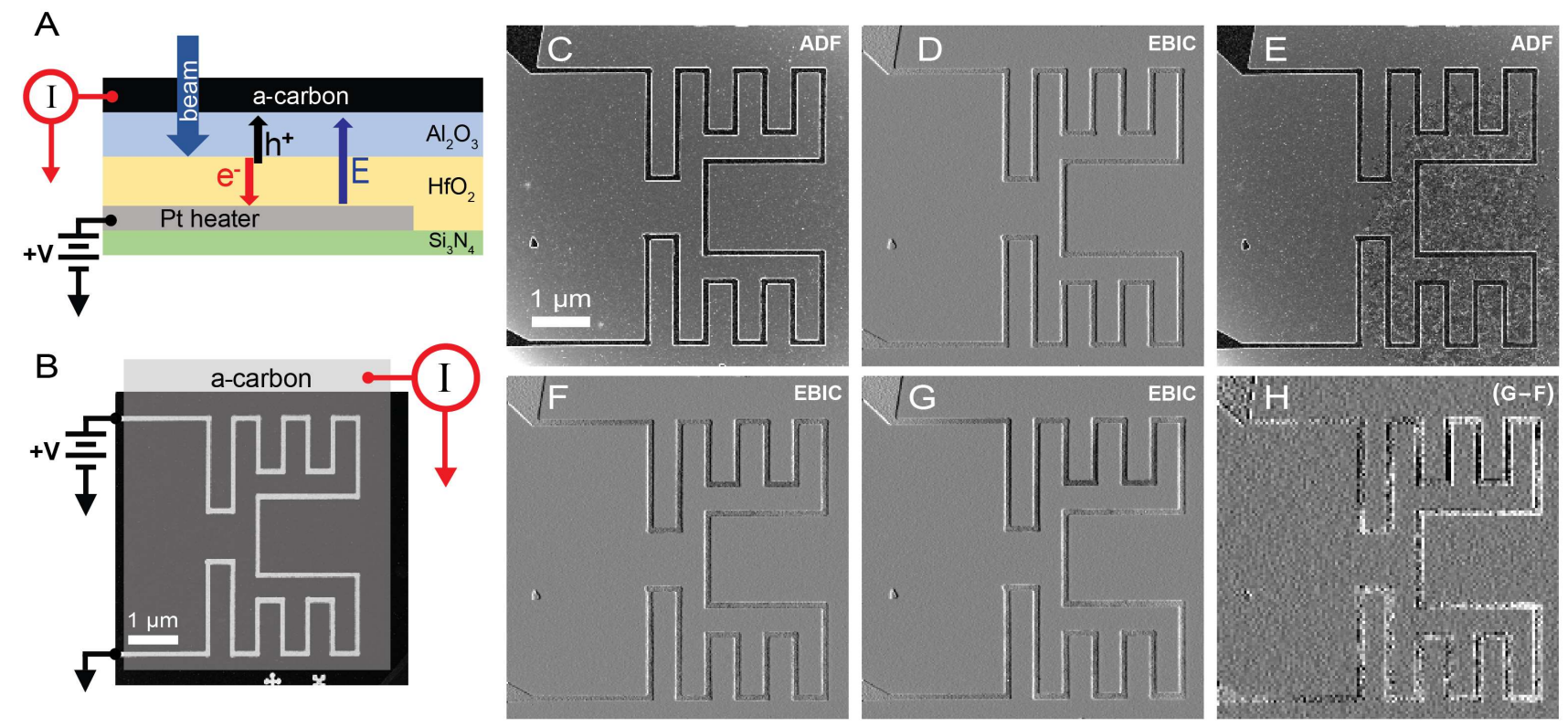

Figure 1. Device diagram (A-B), ADF STEM, and EBIC data showing evidence of ferroelectricity in $\mathrm{HfO}_{2}(\mathrm{C}-\mathrm{F})$. The device, fabricated on an electron transparent $\mathrm{Si}_{3} \mathrm{~N}_{4}$ membrane, consists of a heater coated with thin ALD films of $\mathrm{HfO}_{2}$ and $\mathrm{Al}_{2} \mathrm{O}_{3}$, which are covered with a carbon top electrode. To heat the film, a bias voltage is applied to one side of the heater with the other side grounded. To polarize the $\mathrm{HfO}_{2}$ film, the bias voltage is applied both sides of the heater. The EBIC is measured in the carbon top electrode. Prior to heating, the ALD films show uniform contrast when imaged with STEM ADF (C). After heating, the ADF (E) image shows crystallization of the hafnia near the heater's hot spot. Between the acquisition of EBIC images $(\mathrm{F})$ and $(\mathrm{G})$, a polarizing $2 \mathrm{~V}$ bias (no current) was applied to the heater. In $(\mathrm{H})$, the difference between $(\mathrm{G})$ and $(\mathrm{F})$ binned by 2, a bright EBIC signal in the region of recrystallized hafnia appears, indicating that a positive electric field that persisted after the $2 \mathrm{~V}$ bias voltage was removed. (CD) and (E-F) were acquired simultaneously, and the bias voltage was removed before any of these images were captured. 